This conic is tangent to the curve at $t=0, t=\infty$, and intersects the curve at six other points. At one of the latter points a tangent to the conic is tangent to the curve at some other point. We may summarize with this theorem: The self-dual plane rational quintic admitting of the greatest possible number of correlations is invariant under a $G_{12}$ consisting of collineations and correlations.

Throop College, February, 1919.

\title{
GROUPS CONTAINING A RELATIVELY LARGE NUMBER OF OPERATORS OF ORDER TWO.
}

\author{
BY PROFESSOR G. A. MILLER.
}

(Read before the American Mathematical Society March 29, 1919.)

\section{§1. Introduction.}

IT is well known that every group which contains at least one operator of order 2 must contain an odd number of such operators and that there is an infinite number of groups such that each of them contains exactly $2 m+1$ operators of order 2 , where $m$ is an arbitrary positive integer or 0 . It is also known that if exactly one half of the operators of a group are of order 2 then the order of this group must be of the form $2(2 m+1)$ and it must be the dihedral or the generalized dihedral group of this order. Moreover, it has been proved that a group $G$ of order

$$
g=2^{a}(2 m+1)
$$

cannot contain more than $2^{a} m+2^{a}-1$ operators of order 2 , $\alpha$ being an arbitrary positive integer, and whenever $G$ contains this number of operators of order 2 it is either the abelian group of order $2^{a}$ and of type $(1,1,1, \cdots)$ or it is the direct product of the abelian group of order $2^{a-1}$ and of type (1, 1 , $1, \cdots)$ and the dihedral or the generalized dihedral group of order $2(2 m+1)$.* $^{*}$

* G. A. Miller, this Bulletin, vol. 13 (1907), p. 235. 
From these theorems it results directly that when the order of a group is given but no other restrictions are imposed on the group, it is always possible to find an integer which represents the upper limit for the number of operators of order 2 contained in a group of this order, and also to state how many groups of this order contain this number of operators of order 2 . In particular, there is no group of order 1,000 which contains more than

$$
2^{3} \cdot 62+2^{3}-1=503
$$

operators of order 2 , and there are exactly three groups of order 1,000 which contain separately 503 operators of this order since there are three abelian groups of order $p^{3}, p$ being a prime number.

When the number of operators of order 2 contained in $G$ exceeds $g / 2$, this excess cannot be an even number, for if it were an even number $2 m$ it would result that the order of $G$ would have to be twice an odd number. In fact, if we let $2 k$ represent the number of the operators of order greater than 2 in such a group, it would follow that

$$
2 k+2 m+1=g / 2 .
$$

Since a group whose order is twice an odd number contains a subgroup of half its order composed of its operators of odd order, it results that $m=0$ whenever $2 m$ represents the number of the operators of order 2 in excess of half the order of the group. That is, whenever more than half of the operators of a group are of order 2 this excess is an odd number. This elementary theorem will be generalized in the following section.

Let $g / 2-k, k$ being a positive integer, represent the number of the operators of order 2 contained in $G$. When $k$ is even, $g$ is of the form $2(2 m+1)$ and hence $G$ contains a subgroup of order $2 m+1$. If $t$ represents any operator of order 2 contained in $G$, the product of $t$ and an operator in this subgroup of order $2 m+1$ cannot be of order 2 unless $t$ transforms this operator into it's inverse. As all of the operators of a group must correspond to their inverses whenever more than three-fourths of them correspond to their inverses in an automorphism of the group, it results that when $k$ is even $2 m+1 \leqq 4 k$. In particular, there is only a finite number of groups which satisfy the condition that the number of their operators of order 2 is equal to half the order of the group minus a given even number. 
While the number of the operators of order 2 contained in a group cannot be equal to one half of the order of the group plus a positive even number, it can be equal to one-half the order of the group minus an arbitrary positive number. In fact, there is a cyclic group such that the number of its operators of order 2 is $g / 2-k, k$ being an arbitrary positive integer. The order of this cyclic group is clearly $2(k+1)$. When $k=2$ or 4 there is no other group satisfying the given condition, but when $k=6$ there is also a non-cyclic group of order 18 which involves exactly $9-6=3$ operators of order 2 , as can easily be verified.

\section{§2. Groups in Which More Than One-Half of the. Operators are of Order Two.}

Whenever more than one half of the operators of $G$ are of order 2, this excess must be an odd number, as was noted above. We shall now prove that this odd number is always of the form $2^{a}-1$. When $G$ is abelian and of type $(1,1,1$, ...) it is evident that this condition is satisfied. In all other cases $G$ contains a non-invariant operator $s_{1}$ of order 2 . Let $H_{1}$ represent the subgroup of $G$ composed of all the operators of $G$ which are commutative with $s_{1}$ and let $G-H_{1}$ represent the totality of the operators of $G$ which are not contained in $H_{1}$. Since each of the operators of $G-H_{1}$ is non-commutative with $s_{1}$ it results that at least one half of these operators have orders which exceed 2 , and hence more than one half of the operators of $H_{1}$ are of order 2.

When $H_{1}$ is abelian it must be of order $2^{n}$ and of type $(1,1,1, \cdots)$. If it is non-abelian, it contains a non-invariant operator $s_{2}$ of order 2 , and we let $H_{2}$ represent the subgroup composed of all the operators of $H_{1}$ which are commutative with $s_{2}$. The totality of operators $H_{1}-H_{2}$ will again contain at least as many operators whose orders exceed 2 as the number of its operators of order 2, and the central of $\mathrm{H}_{2}$ must exceed that of $H_{1}$, which, in turn, exceeds that of $G$. By continuing this process we must arrive at an abelian group $H_{m}$ composed of all the operators of $H_{m-1}$ which are commutative with one of its non-invariant operators $s_{m}$ of order 2. The subgroup $H_{m}$ has an order of the form $2^{n}$ and is of type $(1,1,1, \cdots)$.

Since $H_{m}$ is a subgroup of $G$ it is well known that all the operators of $G$ may be uniquely represented as follows:

$$
H_{m}+H_{m} t_{2}+H_{m} t_{3}+\cdots+H_{m} t_{\gamma} .
$$


As $H_{m}$ contains each of the operators $s_{1}, s_{2}, \cdots, s_{m}$, it is evident that at least half of the operators in each of these co-sets have orders which exceed 2 . On the other hand, there is at most one of the co-sets $H_{m} t_{2}, H_{m} t_{3}, \cdots, H_{m} t_{\gamma}$ in which the number of operators whose orders exceeds 2 is larger than the number of its operators of order 2 . To prove this fact it is only necessary to observe that the number of operators of order 2 in $H t_{\alpha}, 2 \leqq \alpha \leqq \gamma$, is equal to the order of the subgroup of $H_{m}$ composed of its operators which are commutative with $t_{a}$. If this order were less than $2^{n-1}$ for two values of $\alpha$ the number of operators of order 2 contained in $G$ would be less than $g / 2$, $2^{n}$ being the order of $H_{m}$.

If the number of operators of order 2 in each of the co-sets $H_{m} t_{2}, H_{m} t_{3}, \cdots, H_{m} t_{\gamma}$ is equal to $2^{n-1}$ then $2^{n-1}$ represents also the excess over $g / 2$ of the number of the operators of order 2 contained in $G$. If one of these co-sets $H t_{a}$ contains more operators whose orders exceed 2 than operators of order 2 this excess is equal to the number of operators of order 2 which are both contained in $H_{m}$ and commutative with $t_{a}$. Hence it has been proved that whenever the number of the operators of order 2 contained in a group exceeds one half of the order of the group this excess must be of the form $2^{a}-1$.

From the theorem which has just been proved it is easy to find the form of all the possible ratios between $g$ and the number of operators in $G$ whose orders exceed 2 whenever $g$ is of the form $2^{k}$. In fact, this number is evidently $2^{k-1}-2^{a}$ and hence this ratio is always of the form

$$
\frac{2^{k}}{2^{k-1}-2^{\alpha}}=\frac{2^{\beta}}{2^{\beta-1}-1} .
$$

Moreover, there is an infinite system of such groups for every positive integral value of $\beta>1$. $^{*}$ It may also be noted that whenever one of the co-sets $H t_{a}, 2 \leqq \alpha \leqq \gamma$, involves more operators whose orders exceed 2 than operators of order 2 , this co-set is composed of all the operators of $G$ which are commutative with less than one half of the operators of $H_{m}$. Hence this co-set involves the inverses of all its operators and therefore each of its operators transforms $H_{m}$ into itself. As one of these operators is of order 2 this co-set and $H_{m}$ generate a group whose order is twice the order of $H_{m}$, and hence the order of each operator of this co-set is a divisor of 4 .

\footnotetext{
* G. A. Miller, Annals of Mathematics, vol. 7 (1906), p. 57.
} 
We proceed to prove that whenever $g$ is not of the form $2^{\alpha}$ then exactly one half of the operators in each of the co-sets $H t_{\alpha}, 2 \leqq \alpha \leqq \gamma$, are of order 2 . If this condition were not satisfied, $H_{m}$ would involve an operator $s_{1}$ which would be commutative with an operator $t$ of odd order contained in $G$ and would transform into its inverse an operator $t^{\prime}$ of order 4 found in the co-set in which more than half the operators would be of order 4 . This follows directly from the fact that all the operators of the co-set involving $t$ are commutative with exactly half the operators of $H_{m}$ while $t^{\prime}$ is commutative with at most one fourth of the operators of this subgroup.

Let $H_{1}$ be the subgroup composed of all the operators of $G$ which are commutative with $s_{1}$. If the product of $t^{\prime}$ and an operator $t_{1}{ }^{\prime}$ of order 2 which is found in $H_{1}$ but not in $H_{m}$ had an order larger than 2 then $t_{1}^{\prime}$ and $t^{\prime}$ would be commutative since this product would be transformed into its inverse by $s_{1}$ and hence $\left(t^{\prime} t_{1}\right)^{-1}=t^{\prime-1} t_{1}{ }^{\prime}$. Therefore, it results that $t^{\prime}$ is transformed either into itself or into its inverse by all the operators of the group generated by the operators of order 2 found in $H_{1}$ but not in $H_{m}$. These operators clearly generate $H_{1}$, since $H_{1}$ involves an operator of odd order and this operator must be contained in each of its subgroups of index 2 .

Since $t$ could not transform $t^{\prime}$ into its inverse it follows that $t$ and $t^{\prime}$ are commutative. Their product must be transformed into its inverse by $s_{1}$ and hence we are led to the contradictory equation

$$
\left(t^{\prime} t\right)^{-1}=t t^{\prime-1} \text {. }
$$

As the assumptions that one of the given co-sets contains more operators of order 4 than of order 2 and that the order of $G$ is divisible by an odd prime number led to a contradiction, we have proved that exactly half of the operators of each of these co-sets must be of order 2 whenever $g$ is divisible by an odd prime.

It will now be proved that the subgroup of index 2 under $H_{m}$ composed of all the operators of $H_{m}$ which are commutative with $t_{\alpha}$ is the same for every value of $\alpha$ from 2 to $\gamma$. If this were not true, the subgroup formed by the operators of $H_{m}$ which are commutative with $t$ would involve an operator $s_{1}$ which would transform into its inverse an operator $t_{1}$ not found in the subgroup $H_{1}$ composed of all the operators of $G$ which are commutative with $s_{1}$. Just as before, we may 
prove that $t_{1}$ is commutative with $t$ because it is transformed either into itself or into its inverse by all the operators of order 2 contained in $H_{1}$ but not in $H_{m}$.

Moreover, $t t_{1}$ is transformed into $t_{1}^{-1} t^{-1}=t^{-1} t_{1}^{-1}$ whenever the order of this product exceeds 2 . If this order were $2, s_{1}$ would transform $\left(t t_{1}\right)^{2}=t^{2} t_{1}^{2}$ into $t^{2} t_{1}{ }^{-2}=t^{2} t_{1}{ }^{2}$. As this leads to a contradiction and as $t t_{1}$ is also transformed into $t t_{1}^{-1}$ by $s_{1}$, it results that the two assumptions that $G$ contains operators of odd order and that some of the operators of $H_{m}$ which are commutative with a certain $t_{\alpha}$ are not commutative with every $t_{\alpha}, 2 \leqq \alpha \leqq \gamma$, are contradictory. It therefore results that exactly $\bar{h}$ alf of the operators of $H_{m}$ constitute the central of $G$ whenever $g$ is divisible by an odd prime number.

Let $K$ represent the central of $G$ and suppose that $g$ is divisible by an odd prime number. The quotient group $G / K$ has an order which is divisible by all the odd divisors of $g$ and at least one half of its operators are of order 2 . If exactly half of these operators are of order 2, this quotient group is either the dihedral or the generalized dihedral group whose order is of the form $2(2 m+1)$. If more than one half of its operators are of order 2 , we may proceed as above and find a second quotient group in which at least one half of the operators are of order 2. Hence we have established the following theorem: If the order of a group is $2^{a}(2 m+1), m>0$, and if more than one half of its operators are of order 2 then this group contains an invariant subgroup of order $2^{a-1}$ and the corresponding quotient group is either the dihedral or the generalized dihedral group of order $2(2 m+1)$.

The subgroup of $G$ which corresponds to the subgroup of order $2 m+1$ in the quotient group does not involve any of the operators of $H_{m}$ which transform each of the operators of $G$ whose orders exceed 2 into their inverses, since more than one half of the operators of the former subgroup have orders greater than 2. This subgroup must be abelian since all of its operators whose orders exceed 2 correspond to their inverses in an automorphism and the products of these operators must also correspond to their inverses. It therefore results from the preceding theorem that, if a group whose order is divisible by an odd prime number has the property that at lecist one half of its operators are of order 2 , it is either a dihedral or a generalized dihedral group. It also results that $H_{m}$ is identical with $H_{1}$ whenever $g$ has an odd prime factor but this is not necessarily true when $g$ is of the form $2^{a}$, as can easily be verified. 